\title{
Construção e validação de um questionário para identificação da Síndrome de Ansiedade de Separação em cães domésticos
}

\author{
Development and Validation of a questionnaire to identify Separation Anxiety Syndrome \\ in domestic dogs
}

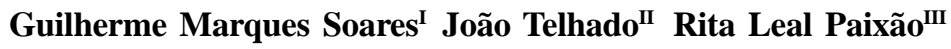

\section{RESUMO}

Em cães, a Síndrome de Ansiedade de Separação em Animais (SASA) pode ser definida como um conjunto de comportamentos indesejáveis exibidos por esses animais quando afastados da figura de apego. A partir da necessidade de um levantamento epidemiológico sobre esse problema, foi desenvolvido um questionário para identificação da SASA em cães (QI-SASA) com base em dados da literatura e validado por meio de dois procedimentos. O primeiro foi a comparação entre pareceres de médicos veterinários brasileiros que atuam na área de etologia clínica a respeito de 40 QI-SASA respondidos por proprietários de cães. O segundo foi a comparação dos resultados da avaliação de 40 QI-SASA, a partir dos critérios estabelecidos neste trabalho, com o resultado de entrevistas para o diagnóstico da SASA. O QI-SASA mostrouse completo, coerente e confiável com a finalidade de um levantamento epidemiológico do problema.

Palavras-chave: ansiedade de separação, cães, comportamento, bem-estar animal.

\section{ABSTRACT}

The Separation Anxiety Syndrome in Animals (SASA) and being more specific, in dogs, can be defined as a group of undesirable behaviors displayed by dogs when they are away from the attachment subject. The questionnaire was developed due to the need to identify this disorder and conduct an epidemiologic survey on SASA in dogs (QI-SASA). This instrument was developed based on reference data and was validated through two procedures. The first one was the comparison among evaluations of Brazilian ethologists on the appliance of 40 QI-SASA to dogs' owners. The second was the comparison between the previous evaluations, according to the criteria established in this research, with the results of the interviews to diagnose SASA. The QI-SASA seems to be a complete, safe and trustworthy tool to carry out epidemiologic surveys on SASA.

Key words: separation anxiety, dogs, behavior, animal welfare.

\section{INTRODUÇÃO}

Na clínica médica de pequenos animais, freqüentemente há relatos de proprietários sobre a grande dificuldade que encontram em deixar seus cães sozinhos em casa. Discute-se também o forte impacto que tal fato causa em sua qualidade de vida devido à freqüente preocupação com o cão, com a destruição que provoca quando fica só e com a queixa de vizinhos por causa dos latidos. Tal contexto geralmente está associado a um cão com Síndrome de Ansiedade de Separação em Animais (SASA).

Em cães, a SASA compreende um conjunto de comportamentos exibidos por esses animais quando são afastados fisicamente de seus proprietários ou de outras figuras de apego, freqüentemente descrito quando esses animais são deixados sozinhos em casa. Os comportamentos descritos por vários autores

'Programa de Pós-graduação em Medicina Veterinária, Clínica e Reprodução Animal, Faculdade de Veterinária, Universidade Federal Fluminense (UFF). Rua Noronha Torrezão, 282/902, Bl 01, 24240-182, Santa Rosa, Niterói, RJ, Brasil. E-mail: gsoaresvet@oi.com.br. Autor para correspondência.

"Departamento de Medicina e Cirurgia Veterinária, Instituto de Veterinária, Universidade Federal Rural do Rio de Janeiro (UFRRJ), Seropédica, RJ, Brasil.

"I'Departamento de Fisiologia e Farmacologia, Instituto Biomédico, UFF, Niterói, RJ, Brasil. 
(MCCRAVE, 1991; FOGLE, 1992; OVERALL, 1997; KING et al., 2000; BEAVER, 2001; APPLEBY \& PLUIJMAKERS, 2003; BENEZECH, 2003; SCHWARTZ, 2003; LANDSBERG et al., 2004; SOARES et al., 2007) são vocalização excessiva, destruição de objetos, micção e defecação fora do lugar determinado. A síndrome também pode incluir vômitos e depressão (MCCRAVE, 1991; FOGLE, 1992; BENEZECH, 2003; LANDSBERG et al., 2004), além de comportamentos compulsivos como a tricotilomania ou a lambedura compulsiva de membros ou flanco (MCCRAVE, 1991; FOGLE, 1992; BEAVER, 2001; OVERALL \& DUNHAM, 2002; LANDSBERG et al., 2004). ASASA também pode ser definida como apreensão decorrente da remoção de pessoas vinculadas ao cão ou de ambientes familiares (APPLEBY \& PLUIJMAKERS, 2003).

O cão pode manifestar os sinais da SASA mesmo quando não está sozinho, pois o animal pode se vincular a um único indivíduo canino ou humano (MCCRAVE, 1991; APPLEBY \& PLUIJMAKERS, 2003; LANDSBERG et al., 2004) e, na ausência da figura de vínculo, iniciam-se os sinais. Tal fato explica o aparecimento da SASA em cães, em abrigos e em residências com várias pessoas e animais, embora cães que vivam com uma só pessoa demonstrem maior predisposição para o distúrbio (SCHWARTZ, 2003). A síndrome pode se manifestar quando o cão tem restrito, de alguma maneira, o acesso à figura de vínculo (contido em algum cômodo, caixa de transporte, gaiola), ainda que esta esteja na mesma casa e há poucos metros do animal (MCCRAVE, 1991; LANDSBERG et al., 2004).

Os quatro sinais básicos (comportamentos destrutivos, vocalização excessiva, defecação e micção inapropriadas) podem ser explicados como tentativas de restabelecer contato com sua matilha ausente, como ocorre com a vocalização excessiva e os comportamentos destrutivos. Os últimos normalmente são direcionados a pertences do proprietário ou a portas, janelas ou móveis que possam ser usados como rotas de fuga pelo cão. Os casos que apresentam distúrbios de eliminação podem ser considerados mais graves, pois a micção e a defecação já caracterizam uma perda do controle da situação por parte do cão, excedendo uma possível estratégia para estabelecer contato (APPLEBY \& PLUIJMAKERS, 2003).

Estudiosos do comportamento animal (MCCRAVE, 1991; KING et al., 2000; APPLEBY \& PLUIJMAKERS, 2003; BENEZECH, 2003; LANDSBERG et al., 2004) sugerem que a hipervinculação é uma condição não só predisponente, mas também necessária para a SASA. Para MCGRAVE (1991), a hipervinculação é a chave para diferenciar os comportamentos indesejáveis apresentados na SASA de outras possíveis origens, tais como marcação territorial, manifestações extremas de submissão, falta de “educação sanitária”, mastigação infantil, hiperatividade, resposta a estímulos externos (pessoas fora da casa, outros cães latindo ou uivando), brincadeiras e respostas agressivas ou induzidas pelo medo.

A manifestação típica de hipervinculação é a organização de todas as atividades do cão em torno da figura de vínculo, quando esse indivíduo está presente. Tais atividades do animal incluem seguir o dono cômodo a cômodo, não deixando que este vá ao banheiro sem que o cão o espere, esperar que o indivíduo durma e inclinar-se sobre ele e demonstrarse ansioso quando separado do dono, mesmo este estando em casa, o que pode envolver destruição da via de acesso. Em relação à população canina de forma geral, tais cães também exageram em relação aos cumprimentos efusivos quando seus proprietários retornam a casa (APPLEBY \& PLUIJMAKERS, 2003; LANDSBERG et al., 2004).

Cães hipervinculados ao proprietário começam a apresentar sinais da SASA quando este se prepara para sair, pois conhecem a rotina e antecipam sua saída. Esses sinais podem variar de tentativas de impedir a partida do dono, tremores, "sapateios” e agitação de maneira geral, até comportamentos depressivos (MCCRAVE, 1991; LANDSBERG et al., 2004). Os comportamentos depressivos normalmente são identificados em relatos de proprietários em que o cão, quando o dono se prepara para sair, vai para um local específico e lá fica quieto até o seu retorno, sem comer, beber, urinar nem defecar.

Cerca de $14 \%$ dos cães atendidos por médicos veterinários dos Estados Unidos da América (OVERALL, 2001; DENENBERG et al.., 2005), do Canadá e da Austrália (DENENBERG et al., 2005) apresentam sinais de SASA, podendo esse percentual alcançar 40\% (SEKSEL \& LINDEMAN, 2001). Este é um dos três problemas de comportamento mais freqüentes apresentados na Clínica de Comportamento Animal da Universidade de Cornell (EUA), juntamente com agressões aos proprietários e/ou a estranhos (TAKEUCHI et al., 2001). No Brasil, não há estudos que revelem um registro nacional sobre a ocorrência de SASA, mas são freqüentes relatos condizentes com a síndrome na clínica médica de pequenos animais.

O objetivo deste estudo é descrever o processo de elaboração de um questionário para detecção de comportamentos sugestivos de SASA em cães domésticos a fim de que esse instrumento seja útil para realização de um levantamento epidemiológico da SASA. 


\section{MATERIAL E MÉTODOS}

Para guiar o processo de construção e validação do questionário para identificação da SASA em cães (QI-SASA), foram realizadas as seguintes etapas:

fase 1 - planejamento- Nessa fase, foram definidos o objetivo, o tipo de população-alvo, os tipos de itens e o formato do questionário. Essa etapa foi inicialmente sustentada na extensa revisão de literatura realizada a partir de consulta às publicações acerca do tema. Buscou-se localizar instrumentos utilizados para identificação de SAS e constatou-se sua inexistência na literatura internacional e nacional. Optou-se pelo formato de questionário destinado aos proprietários, pois estes são considerados as melhores fontes de dados epidemiológicos relacionados a problemas comportamentais (FATJÓ et al., 2006);

fase 2 - elaboração do questionário - a versão final do questionário (o questionário em português fica disponível a partir de contato com os autores) constituiu-se de 19 perguntas semi-abertas que abordavam aspectos de diferentes domínios do comportamento animal discutidos na literatura e que visavam identificar: (1) sinais de SASA e (2) sinais de hipervinculação. As perguntas deveriam ser respondidas de acordo com as orientações gerais no cabeçalho do questionário, as quais indicavam: marcar quantos itens considerasse necessários para qualificar o comportamento de seu cão; responder apenas sobre um cão, caso possuísse mais de um em casa, e definir como proprietário a pessoa de quem o cão mais gostasse. Os itens/as perguntas foram agrupados em diferentes domínios (vocalização excessiva, comportamento destrutivo, eliminações inapropriadas, alterações autonômicas, depressão e comportamentos compulsivos) para verificar a presença ou ausência dos sinais de SASA e avaliação de hipervinculação (Tabela 1). Todos os itens tinham uma opção aberta para o respondente adicionar opções que não estivessem listadas. Foram listados outros comportamentos para não permitir que o respondente identificasse o objetivo das perguntas, a fim de que as respostas não fossem direcionadas de maneira a enquadrar ou não seu cão no problema pesquisado. A última questão do questionário foi inserida a fim de descartar a inclusão de animais com outros distúrbios que possam afetar o comportamento;

fase 3 - exame subjetivo de conteúdo - depois de elaborado, o questionário foi avaliado por um grupo de médicos veterinários, estudantes do Programa de Pós-graduação em Medicina Veterinária, área de concentração em Clínica e Reprodução Animal da
Universidade Federal Fluminense. Em seguida, o questionário foi avaliado por pessoas leigas no assunto para avaliar se a terminologia e abrangência eram suficientes para caracterizar o problema quando respondido por pessoas leigas. O questionário foi analisado por três especialistas na área de comportamento animal para avaliar se todos os aspectos da SASA estavam contemplados. Também foi realizada uma pesquisa-piloto com seis proprietários de cães que não conheciam os objetivos do instrumento para avaliar a clareza da terminologia empregada, a extensão do questionário e o tempo gasto para respondê-lo, para garantir a viabilidade de todo o processo.

\section{Critérios de Avaliação do QI-SASA}

Para fins deste estudo, foram considerados como positivos para a SASA os animais que apresentavam ao menos qualquer um dos sinais clássicos (vocalização excessiva, comportamento destrutivo, micção inapropriada e defecação inapropriada) ou comportamento depressivo, isolado ou combinado, associado a, pelo menos, três opções relacionadas à hipervinculação (Tabela 1 ). O quadro depressivo só foi caracterizado a partir de três opções marcadas no mesmo questionário: (1) o cão não come quando fica sozinho; (2) já se mostrou depressivo na ausência de algum familiar; e (3) se desloca para um local específico isolado no momento da partida do proprietário;

fase 4 - validação do QI-SASA - nessa etapa, o questionário foi respondido por um número significativo de indivíduos (40 proprietários de animais) para exame da confiabilidade e validade do instrumento criado, utilizando-se dois diferentes procedimentos.

\section{Primeiro procedimento de validação}

Nessa etapa, os 40 questionários aplicados (18 respondidos por proprietários de cães de apartamento, em Niterói, Rio de Janeiro, e 22 respondidos por proprietários de cães que são alunos da Universidade Federal Rural do Rio de Janeiro) foram avaliados de forma independente por quatro médicos veterinários atuantes na área de etologia clínica. Os resultados foram comparados dois a dois, agrupados em tabelas 2 X 2 e avaliados estatisticamente. Também foi feita uma comparação entre a concordância de dois pareceres dos Avaliadores 2, 3 e 4 com o parecer do Avaliador 1,obtido por meio dos critérios de avaliação descritos neste trabalho.

Segundo procedimento de validação

O segundo procedimento teve por objetivo comparar os resultados do QI-SASA com a forma de 
Tabela 1 - Relação de comportamentos abordados no Questionário para Identificação da Síndrome de Ansiedade de Separação em Animais com sua distribuição em opções por itens, Universidade Federal Fluminense, Niterói, 2007.

\begin{tabular}{|c|c|c|}
\hline Sinais & Item/pergunta & opção \\
\hline \multirow{4}{*}{ Vocalização Excessiva } & \multirow{2}{*}{ Quando late? } & ao ficar preso \\
\hline & & ao ficar sozinho \\
\hline & Quando fica sozinho & chora \\
\hline & Outros comportamentos & chora ou uiva quando fica preso \\
\hline \multirow{4}{*}{$\begin{array}{l}\text { Comportamento } \\
\text { Destrutivo }\end{array}$} & Em relação a pertences das pessoas da casa & destrói na sua ausência \\
\hline & \multirow{3}{*}{ Outros comportamentos } & arranha portas/janelas quando fica sozinho \\
\hline & & arranha portas/janelas quando fica preso \\
\hline & & $\begin{array}{l}\text { arranha móveis próximo às janelas externas quando fica sozinho } \\
\text { arranha o chão quando fica sozinho ou preso }\end{array}$ \\
\hline \multirow{2}{*}{$\begin{array}{l}\text { Eliminações } \\
\text { Inapropriadas }\end{array}$} & Onde urina? & em lugares inapropriados da casa, quando fica sozinho ou preso \\
\hline & Onde defeca? & em lugares inapropriados da casa, quando fica sozinho ou preso \\
\hline \multirow{3}{*}{$\begin{array}{l}\text { Alterações } \\
\text { Autonômicas }\end{array}$} & Quando fica sozinho & vomita \\
\hline & Ouande o pronriotário se prenara nara cair & fica salivando \\
\hline & Quando o proprletarıo se prepara para sair & fica ofegante \\
\hline \multirow{3}{*}{ Depressão } & Quando o proprietário se prepara para sair & vai para um “cantinho" e fica quieto \\
\hline & Quando fica sozinho & não come \\
\hline & Outros comportamentos & $\begin{array}{l}\text { "já se demonstrou triste ou depressivo na ausência de algum } \\
\text { membro da família }\end{array}$ \\
\hline \multirow{10}{*}{ Hipervinculação } & Quando o proprietário chega em casa & $\begin{array}{l}\text { faz festa calorosamente } \\
\text { faz festa de forma exagerada }\end{array}$ \\
\hline & $\begin{array}{l}\text { Quando outras pessoas da família chegam } \\
\text { em casa }\end{array}$ & $\begin{array}{l}\text { faz festa calorosamente } \\
\text { faz festa de forma exagerada }\end{array}$ \\
\hline & & mostra-se agressivo \\
\hline & & mostra-se agitado \\
\hline & Ouando o nronrietário se prenara nara sair & fica ofegante \\
\hline & Quando o proprletarıo se prepara para sair & fica salivando \\
\hline & & vai para um “cantinho" e fica quieto \\
\hline & & tenta impedir de alguma forma. Como? \\
\hline & & segue o proprietário pela casa tentando estar sempre perto \\
\hline & Outros comportamentos & $\begin{array}{l}\text { mostra-se agitado quando afastado poucos metros da pessoa de } \\
\text { quem ele(a) mais gosta }\end{array}$ \\
\hline \multirow{4}{*}{$\begin{array}{l}\text { Comportamentos } \\
\text { Compulsivos }\end{array}$} & Outros comportamentos & caça moscas imaginárias \\
\hline & & as patas com muita freqüência \\
\hline & Tem hábito de lamber... & outra parte do corpo com muita freqüência \\
\hline & & com freqüência algum lugar ou objeto da casa \\
\hline
\end{tabular}

obtenção de dados normalmente empregada na rotina clínica para o diagnóstico da SASA, que é a anamnese. Para tanto, foram realizadas 40 entrevistas em conjunto com a aplicação dos questionários, de forma aleatória, com proprietários de cães no Hospital Universitário Veterinário da Universidade Federal Fluminense.
Participaram dessa etapa apenas aqueles proprietários que não haviam participado de nenhuma etapa do estudo. Essas entrevistas foram desenvolvidas como uma anamnese para a identificação da SASA. Os QISASA foram examinados de maneira que o avaliador não tivesse acesso a nenhuma informação sobre os 
questionários, além das necessárias para a avaliação. As entrevistas foram analisadas independentemente dos questionários e também foram caracterizadas como "positivas” ou "negativas” para SASA. Os resultados das entrevistas foram agrupados e comparados aos resultados da análise dos QI-SASA. Os dados foram tabulados e tratados estatisticamente de acordo com o procedimento anterior.

\section{Cálculos estatísticos}

Para as comparações entre os pareceres dos questionários QI-SASA e entre os resultados dos QISASA e das entrevistas, foram realizados o teste de Mc Nemar $(\chi \mathrm{mc})$ e o cálculo do coeficiente Kappa (k). Os dois testes têm a função de avaliar a correlação entre pareceres. O teste de Mc Nemar determina se há ou não concordância entre os grupos, ou seja, se estatisticamente os dois grupos são iguais ou diferentes (VIEIRA, 2003; ARANGO, 2005). Já o coeficiente Kappa determina o quanto os dois grupos de pareceres concordam entre si (PERROCA \& GAIDZINSKI, 2003; ARANGO, 2005). Todos os testes foram realizados de acordo com ARANGO (2005) ou por meio do programa Graphad Instat ${ }^{\circledR}$ V.2.0. O nível de significância para todos os testes aplicados foi de 5\% $(\alpha=0,05)$.

\section{RESULTADOS E DISCUSSÃO}

Exame subjetivo de conteúdo - O grupo de médicos veterinários e os demais profissionais que participaram do exame subjetivo de conteúdo aprovaram a terminologia em termos de facilidade de entendimento e sugeriram pequenas alterações que foram acatadas. Os médicos veterinários especialistas em comportamento animal também aprovaram o questionário no sentido de abordar todos os aspectos analisáveis da SASA.

A pesquisa-piloto respondida por seis proprietários de cães de apartamento em Niterói apresentou como tempo médio de preenchimento dos questionários 7,8 minutos (desvio padrão=2,14). Dois respondentes do piloto foram interrompidos por eventos externos durante o preenchimento do QISASA, aumentando o tempo para sua conclusão. Todos os respondentes consideraram o questionário claro e simples de ser preenchido.

\section{Validação do questionário}

No primeiro procedimento da validação (Tabela 2), a comparação entre os avaliadores 1 e 2 obteve concordância estatística $\left(\chi^{2} \mathrm{mc}=0,25 ; \mathrm{P}=0,617\right)$ e correlação forte $(k=0,8)$. Já entre os avaliadores 1 e 3, e 2 e 3, que obtiveram os mesmos resultados numéricos, não houve concordância estatística $\left(\chi^{2} \mathrm{mc}=13,06\right.$; $\mathrm{P}<0,001)$ e correlação fraca $(k=0,3)$. Os resultados da comparação dos avaliadores 1 e 2 com o avaliador 4 também não apresentaram concordância estatística $\left(\chi^{2} \mathrm{mc}=5,818 ; \mathrm{P}=0,016\right)$ e correlação moderada $(k=0,5)$. A comparação entre os pareceres dos avaliadores 3 e 4 obteve correlação moderada $(k=0,4)$ e concordância estatística $\left(\chi^{2} \mathrm{mc}=2,50 ; \mathrm{P}=0,114\right)$. A última comparação realizada, entre a concordância de dois resultados entre os avaliadores 2, 3 e 4 com o avaliador 1, que usou os critérios definidos por esta pesquisa, obteve correlação forte $(k=0,8)$ e concordância estatística $\left(\chi^{2} \mathrm{mc}=0,25\right.$; $\mathrm{P}=0,617)$. Comparando os pareceres dos quatro avaliadores em relação aos 40 QI-SASA, 20 resultados foram unânimes e 14 obtiveram pelo menos três concordâncias, chegando a 34 (85\%).

Apesar das diferenças individuais, principalmente entre os avaliadores 1 e 2 com os avaliadores 3 e 4, as coincidências entre os pares, a concordância dos critérios com a maioria e o alto índice de pareceres semelhantes no todo demonstram confiabilidade técnica e coerência na construção do questionário.

O segundo procedimento, que comparou os resultados do QI-SASA com as entrevistas, apresentou correlação estatística entre as duas diferentes abordagens $\left(\chi^{2} \mathrm{mc}=0,071, \mathrm{P}=0,789\right)$, porém fraca concordância $(k=0,3)$.

Vários trabalhos com validações de questionários (FERNANDES \& SOUZA, 2001; PAGANO et al., 2004; SILVA FILHO et al., 2005; FREEMAN, 2005) comparam seus resultados com padrões já pré-existentes, porém não há um padrão oficial para SASA, como há para transtornos psiquiátricos humanos, como, por exemplo, o DSM-IV (Diagnostic and Statistical Manual for Mental Disorders, 4⿳亠口了 versão; KAPLAN et al., 1997). Já direcionado a levantar problemas de comportamento em cães de companhia, outros autores (HSU \& SERPELL, 2003) validam um questionário comparando a avaliação deste com diagnósticos de especialistas em comportamento animal nos Estados Unidos da América. A necessidade de elaborar um questionário surgiu no intuito de possibilitar uma coleta de dados consistente para um levantamento sobre a SASA, além de atender uma das principais demandas das ciências da saúde: a oferta de instrumentos qualificados que auxiliem os clínicos a direcionar suas investigações diagnósticas com maior segurança e forneçam aos pesquisadores recursos metodológicos para o estudo de distúrbios de comportamento (REPPOLD, 2005). A opção por um questionário deu-se por sua praticidade. 
Tabela 2 - Resultados das comparações entre os pareceres de quatro avaliadores sobre os mesmos 40 questionários para identificação da Síndrome de Ansiedade de Separação em Animais (SASA). Niterói - RJ, Universidade Federal Fluminense, 2007.

\begin{tabular}{|c|c|c|c|c|c|c|c|c|c|c|c|c|c|}
\hline & & \multicolumn{3}{|c|}{-------Avaliador 2------- } & \multicolumn{3}{|c|}{-------Avaliador 3------- } & \multicolumn{3}{|c|}{-------Avaliador 4------- } & \multicolumn{3}{|c|}{$\begin{array}{c}2 \text { resultados iguais entre os } \\
\text { Avaliadores } 2 \text {, } 3 \text { e } 4\end{array}$} \\
\hline & & + & - & $p / k$ & + & - & $p / k$ & + & - & $p / k$ & + & - & $p / k$ \\
\hline \multirow{2}{*}{ Avaliador 1} & + & 21 & 2 & \multirow{2}{*}{$0,617 / 0,8$} & 8 & 15 & $<0001<03$ & 13 & 1 & \multirow{2}{*}{$0,016 / 0,5$} & 15 & 0 & \multirow{2}{*}{$0,617 / 0,8$} \\
\hline & - & 2 & 15 & & 0 & 17 & $-0,001 / 0,5$ & 10 & 16 & & 8 & 17 & \\
\hline \multirow{2}{*}{ Avaliador 2} & + & & & & 8 & 15 & \multirow{2}{*}{$<0,001 / 0,3$} & 13 & 1 & \multirow{2}{*}{$0,016 / 0,5$} & & & \\
\hline & - & & & & 0 & 17 & & 10 & 16 & & & & \\
\hline \multirow{2}{*}{ Avaliador 3} & + & & & & & & & 6 & 8 & \multirow{2}{*}{0,114 / 0,4 } & & & \\
\hline & - & & & & & & & 2 & 24 & & & & \\
\hline
\end{tabular}

$p=$ valor $p$ calculado a partir do teste de Mc Nemar $/ k=$ resultado do cálculo do coeficiente Kappa.

+ = Positivo para SASA / - = negativo para SASA.

Os critérios para considerar os questionários positivos são semelhantes aos critérios de inclusão de KING e colaboradores (2000) e estão de acordo com os critérios de diagnóstico diferencial para SASA, estabelecidos por MCGRAVE (1991), acrescidos dos sinais de presença de quadro depressivo.

Os resultados significam que, para uma abordagem epidemiológica, os questionários podem substituir as entrevistas de forma confiável, porém esta substituição perde sua confiabilidade para uma avaliação individual de cada caso.

Não foi feita nenhuma avaliação nos animais por se levar em consideração três fatos: (1) as manifestações da SASA raramente ocorrem na presença de pessoas; (2) a SASA é um distúrbio diagnosticado quase exclusivamente por histórico e anamnese; e (3) não há descrições de alterações metabólicas, bioquímicas ou clinicamente detectáveis nos animais com SASA, alterações que poderiam servir como contraprova.

O QI-SASA não tem a pretensão de ser a contraprova para o diagnóstico da SASA e individualmente não substitui a abordagem clínica pessoal que cada caso merece. O instrumento é um meio de mensurar o problema em um grupo de animais, em situações em que a abordagem individual merecida seja difícil, fornecendo dados para um estudo epidemiológico. Ainda assim, o desenvolvimento de instrumentos de diagnóstico e o conhecimento atual sobre a SASA são insuficientes para mensurar o real sofrimento dos animais ao serem deixados sozinhos por seus proprietários, o que requer mais pesquisas para avaliar o real impacto da síndrome na qualidade de vida desses animais.

\section{CONCLUSÃO}

Por meio dos resultados do presente trabalho, concluiu-se que o instrumento para o diagnóstico da SASA em cães, o QI-SASA, mostrouse completo, coerente e confiável para um levantamento epidemiológico do problema, mas não substitui a abordagem minuciosa e individualizada de cada caso, considerando cada particularidade dos agentes da relação (humanos e caninos).

\section{COMITÊ DE ÉTICA}

O projeto foi submetido e aprovado pelo Comitê de Ética em Pesquisa da Faculdade de Medicina da Universidade Federal Fluminense (CEP CMM/HUAP nº. 149/06). Cada participante, ao ser abordado, assinou um termo de consentimento livre e esclarecido.

\section{REFERÊNCIAS}

APPLEBY, D.; PLUIJMAKERS, J. Separation anxiety in dogs: the function of homeostasis in its development and treatment. Veterinary Clinics of North America: Small Animal Practice, v.33, n.2, p.321-344, 2003.

ARANGO, G.H. Bioestatística: teórica e computacional. 2.ed. Rio de Janeiro: Guanabara Koogan, 2005. 423p.

BEAVER, B.V. Comportamento canino: um guia para veterinários. São Paulo: Roca, 2001. 431p.

BÉNÉZECH, M. L'homme et le chien domestique: une pathologie neuropsychiatrique commune? Annales Médico Psychologique, v.161, n.8, p.569-578, 2003.

DENENBERG, S. et al. A comparison of cases referred to behaviorists in three different countries. In: MILLS, D. et al. Current issues and research in veterinary behavioral medicine: papers presented at the 5 th international veterinary behavior meeting. Indiana: Purdue University, 2005. p.56-62.

FATJÓ, J. et al. The epidemiology of behavioural problems in dogs and cats a survey of veterinary practitioners. Animal Welfare, n.15, p.179-185, 2006. 
FERNANDES, P.T.; SOUZA, E.A.P. Protocolos de investigação de variáveis psicológicas na epilepsia infantil. Psicologia: Teoria e Pesquisa, v.17, n.2, p.195-197, 2001.

FOGLE, B. The dog's mind. London: Pelham Books, 1992. 201p.

FREEMAN, L.M. et al. Development and evaluation of a questionnaire for assessing health-related quality of life in dogs with cardiac disease. Journal of American Veterinary Medical Association, v.226, n.11, p.1864-1868, 2005. Disponível em: http://avmajournals.avma.org/doi/abs/10.2460/ javma.2005.226.1864. Doi: 10.2460/javma.2005.226.1864.

HSU, Y.; SERPELL, J.A. Development and validation of a questionnaire for measuring behavior and temperament traits in pet dogs. Journal of American Veterinary Medical Association, v.223, n.9, p.1293-1300, 2003. Disponível em: ht tp://avmajournals.avma.org/doi/abs/10.2460/ javma.2003.223.1293. Doi: 10.2460/javma.2003.223.1293.

KAPLAN, H.I. et al. Transtornos de ansiedade. In: Compêndio de psiquiatria: ciência do comportamento e psiquiatria clínica. 7.ed. Porto Alegre: Artes Médicas, 1997. p.545-583.

KING, J.N. et al. Treatment of separation anxiety in dogs with clomipramina: results from a prospective, randomized, doubleblind, placebo-controlled, parallel-group, mullticenter clinical trial. Applied Animal Behavior Science, v.67, p.255-275, 2000.

LANDSBERG, G. et al. Problemas comportamentais do cão e do gato. 2.ed. São Paulo: Roca, 2004. 492p.

MCCRAVE, E.A. Diagnostic criteria for separation anxiety in the dog. Veterinary Clinics of North America: Small Animal Practice, v.21, p.247-256, 1991.

OVERALL, K.L. Clinical behavioral medicine for small animals. St. Louis - Missouri: Mosby Year Book, 1997. 544p.

OVERALL, K.L. Pharmacological treatment in behavioural medicine: the importance of neurochemistry, molecular biology and mechanistic hypotheses. Veterinary Journal, v.162, p.9-23, 2001. Disponível em: http://www.sciencedirect.com/ science?_ob=ArticleURL\&_udi=B6WXN-45BC06B$17 \&$ \&_user $=687358 \&$ \&doc $=1 \&$ \& f m t $=\&$ \&_orig = searc h \&_s ort $=$ d \& vi e w = c \&_a c c t = C 000037899 \&_v e r s i o n $=1$ \&_u r l V e r s i o $n=0$ \& userid $=687358 \&$ md5 $=$ a $8659 \mathrm{f} 68 \mathrm{~b} 1 \mathrm{a} 5 \mathrm{bef} 19 \mathrm{f} 08 \mathrm{c} 541 \mathrm{a} 4 \mathrm{aac} 811$. Doi: $10.1053 /$ tvjl.2001.0568.
OVERALL, K.L.; DUNHAM, A. Clinical features and outcome in dogs and cats with obsessive-compulsive disorder: 126 cases. Journal of American Veterinary Medical Association, v.221, n.10, p.1445-1451, 2002. Disponível em: http:// avmajournals.avma.org/doi/abs/10.2460/javma.2002.221.1445. Doi: $10.2460 /$ javma.2002.221.1445.

PAGANO, T. et al. Assesment of anxiety and quality of life in fibrolyalgia patients. São Paulo Medical Journal, v.6, n.122, p.252-258, 2004.

PERROCA, M.G.; GAIDZINSKI, R.R. Avaliando a confiabilidade interavaliadores de um instrumento para classificação de pacientes - coeficiente Kappa. Revista da Escola de Enfermagem -USP, v.37, n.1, p.72-80, 2003.

REPPOLD, C.T. Construção, validação e normatização de uma bateria de cinco escalas para avaliação de ajustamento psicológico em adolescentes. 2005. $334 \mathrm{f}$. Tese (Doutorado em Psicologia) - Instituto de Psicologia, Universidade Federal do Rio Grande do Sul, Rio Grande do Sul.

SCHWARTZ, S. Separation anxiety syndrome in dogs and cats. Journal of American Veterinary Medical Association, v.222, n.11, p.1526-1532, 2003.

SEKSEL, K.; LINDEMAN, M.J. Use of clomipramine in treatment of obsessive-compulsive disorder, separation anxiety and noise phobia in dogs: a preliminary, clinical study. Australian Veterinary Journal, v.79, n.4, p.252-256, 2001. Disponível em: http://www3.interscience.wiley.com/journal/ 120741735/abstract?CRETRY=1\&SRETRY=0. Doi: 10.1111/ j.1751-0813.2001.tb11976.x.

SILVA FILHO, C.R. et al. Sintomas climatéricos e qualidade de vida: validação do questionário da saúde da mulher. Revista de Saúde Pública, v.3, n.39, p.333-339, 2005.

SOARES, G.M. et al. Ansiedade de separação e suas implicações na qualidade de vida de cães domésticos. Revista Clínica Veterinária. v.67, p.76-82, 2007.

TAKEUCHI, Y. et al. Differences in background and outcome of three behavior problems of dogs. Applied Animal Behaviour Science. v.70, p.297-308, 2001. Disponível em: h t t p : / / w w w. s c i e n c e d i r e c t. c o m / science?_ob=ArticleURL\&_udi=B6T48-429Y54G$4 \&$ user $=687358 \&$ \&doc $=18$ \& fmt $=$ \&_orig $=$ search\&_sort $=\mathrm{d} \& \mathrm{~V}$ i e $\mathrm{w}=$ c $\&$ _ a c c t $=$ C $000037899 \&$ _version $=1$ \&_ u r l V e r s i o n $=0$ \&_ u s e r i d $=687$ 358\&md5=f14e3e311b658b2cae87d654644b3c15. Doi: 10.1016/S0168-1591(00)00156-8.

VIEIRA, S. Bioestatística: tópicos avançados. 2.ed. Rio de Janeiro: Elsevier, 2003. 216p. 